\title{
The clinical characteristics and prognosis of ovarian endometrioid carcinoma
}

\author{
Jin $\mathrm{XY}^{1}$, Zhang $\mathrm{WP}^{2}$ and $\mathrm{Wu} \mathrm{MF}^{1 *}$ \\ ${ }^{1}$ Department of Gynecology, Affiliated Women and Children's Hospital of Jiaxing University, China \\ ${ }^{2}$ Department of Anesthesiology, Affiliated Women and Children's Hospital of Jiaxing University, China
}

\begin{abstract}
Objective: To investigate the clinical characteristics and relative factors of ovarian endometrioid carcinoma.

Methods: The clinical data of 36 patients with ovarian endometrioid carcinoma were retrospectively analyzed.

Results: Age of the patients with tumor was relatively young $(61.3 \%<55$ years). Major clinical manifestations are pain, bloating, and abnormal vaginal hemorrhage. Ultrasound examination commonly showed cystic-solid lesion. Five-year survival rate of patients in tutor stage I-II was up to $77.9 \%$, stage III to $36.8 \%$, patients undergoing satisfied cytoreductive surgery to $68.5 \%$, patients with ovarian endometrioid carcinoma without endometrial cancer surgery to $87.4 \%$, and the patients with endometrial cancer surgery to $27.4 \%$.

Conclusion: Prognosis of ovarian endometrioid carcinoma patients undergoing cytoreductive surgery early is favorable, but prognosis of those patients with endometrial cancer is poor.
\end{abstract}

\section{Introduction}

With the progress of pathologic diagnosis technology, more and more patients with ovarian endometrioid carcinoma are detected early. For exploring the diagnosis, treatment and prognostic of ovarian endometrioid carcinoma, the clinical data of 36 patients with ovarian endometrioid carcinoma were analyzed retrospectively.

\section{Material and method}

\section{Subjects}

From Jan1, 2007 to Nov 30, 2016, a retrospective analysis was performed on 36 patients with ovarian endometrioid carcinoma. Clinical data were analyzed and follow-up by telephone were made after operation till Mar 31, 2014, 5 patients were lost to follow-up, with a follow-up rate of $86.1 \%$. Thirty-one patients with ovarian endometrioid carcinoma were included in this study.

\section{Treatment}

Operation: All the 31 patients with ovarian endometrioid carcinoma received operation. The basic pattern of operation is cytoreductive surgery (including removal of total hysterectomy, bilateral adnexectomy, appendix, omentum majus, pelvic metastases, and pelvic lymphadenectomy). Recurrence within six months is considered unsatisfied cytoreductive surgery.

Chemotherapy: 30 cases received additional chemotherapy one or two weeks after operation. Chemotherapy is with TP therapy: carboplatin (CBP) AUC4-5 plus taxol135 mg/m². 1 case gave up for being too old.

\section{Statistics analysis}

Data analysis was performed using the statistical software package SPSS 19.0 (SPSS Inc., Chicago, USA). 5-year survival rate was analyzed with Kaplan-Meier method and comparison of groups was with Chisquare test or Fisher's test. $P$ value $<0.05$ was considered significant.

\section{Results}

\section{Comparison of 5-year survival rate}

Ages: 5-year survival rate of patients aged less than 55 years was $70.4 \%$, while patients aged 55 years or more was $49.6 \%$. There was no significant difference between them. $(P=0.326)$

Tumor stage: 5 -year survival rate of patients in tumor stage I-II from FIGO was $77.9 \%$, patients in tumor stage III was $36.8 \%$, and there was significant difference between them $(P=0.033)$. 5-year survival rate of patients in tumor stage I-II was higher than 5-year survival rate of patients in tumor stage III.

Tumor marker: 5 -year survival rate was $83.3 \%$ when preoperative CA125 < 150u/ml; while 5 -year survival rate was $54.2 \%$ when CA125 $\geq 150 \mathrm{u} / \mathrm{ml}$. No statistical differences were found between groups $(P=0.268)$.

Pathologic type: 5-year survival rate of patients with simple type ovarian endometrioid carcinoma and mixed type ovarian endometrioid carcinoma was $63.3 \%$ and $63.6 \%$ respectively and there was no significant difference between them $(P=0.528)$. 5-year survival rate of patients with endometrial cancer or without was $87.4 \%$ and $27.4 \%$ respectively, with significant difference $(P=0.015)$.

Correspondence to: Wu MF, Department of gynecology, affiliated women and children's hospital of Jiaxing university, China, Tel: +8613757370679 , E-mail: zhang650679@163.com

key words: ovarian tumor, endometrial cancer, survival rate

Received: May 08, 2017; Accepted: June 02, 2017; Published: June 05, 2017 
Operation: 5-year survival rate of patients underwent satisfied cytoreductive surgery and without cytoreductive surgery was $68.5 \%$ and $0.0 \%$ respectively $(P<0.001)$. The patients were divided into two groups according to chemotherapy course (Group 1: chemotherapy course $<6$, group 2: chemotherapy course $\geq 6$ ). 5-year survival rate of group 1 and group 2 was $57.8 \%$ and $75 \%$ respectively. There was no significant difference between them $(P=0.581)$ (Table 1$)$.

\section{Discussion}

\section{Clinical pathological features}

It was reported that the incidence of ovarian endometrioid carcinoma was $3.1 \%$ to $24.4 \%$ in patients with ovarian cancer $[1,2]$. The patients with ovarian endometrioid carcinoma accounted for $3.5 \%$ (36 cases) of the ovarian malignant tumor at the corresponding period in our study. Ovarian endometrioid carcinoma has two pathologic types: endometrial carcinogenesis and epithelial differentiation of germinal epithelium, first reported by Sampson and Santesson. repectively. Pathologic diagnosis need to detect endometriosis ectopic focus location malignant cells or differentiation histology manifestation of ovary cambium epithelia toward endometrium in our hospital. Among the 36 cases, 4 patients with endometriosis were accompany with ovarian endometrioid adenocarcinoma, up to $12.9 \%$, which was consistent with the study of Sainz de la Cuesta R, ranged from $10 \%$ to $28 \%$ [2]. Ratio of malignant transformation from endometriosis to cancer was not high. Erzen et al. [3] thought that $1 / 3$ of the patients with ovarian endometriosis malignant neoplasms had endometriosis history. This report showed that tissue type of canceration of endometriosis was mainly ovarian endometrioid

Table 1. Clinical features of patients

\begin{tabular}{|c|l|l|}
\hline \multicolumn{1}{|c|}{ Item } & \multicolumn{1}{|c|}{ Case (\%) } & P-value \\
\hline Age(year) & & 0.205 \\
\hline$\leq 55$ & $19(61.3)$ & \\
\hline$>55$ & $12(38.7)$ & \\
\hline clinical features & & 0.426 \\
\hline pain, bloating & $25(80.6)$ & \\
\hline vaginal bleeding & $17(54.8)$ & \\
\hline pelvis masses found in physical examination & $4(12.9)$ & \\
\hline Frequent urination after menopause & $1(3.2)$ & \\
\hline preoperative CA125 level (U/L) & & 0.029 \\
\hline$\leq 150$ & $11(35.5)$ & \\
\hline$>150$ & $20(64.5)$ & \\
\hline preoperative ultrasound feature & & 0.651 \\
\hline cystic lesion & $6(19.4)$ & \\
\hline solid lesion & $8(25.8)$ & \\
\hline mixed cystic-solid lesion & $17(54.8)$ & \\
\hline tumor diameter (cm) & & 0.473 \\
\hline$<5$ & $6(22.1)$ & \\
\hline $5-10$ & $15(48.9)$ & \\
\hline$>10$ & $9(29.0)$ & \\
\hline FIGO staging & & \\
\hline Stage I & $10(32.3)$ & \\
\hline Stage II & $9(29.0)$ & \\
\hline Stage III & $12(38.7)$ & \\
\hline histological type & & \\
\hline Simple type endometrioid carcinoma & $18(58.1)$ & \\
\hline Mixed type endometrioid carcinoma & $13(41.9)$ & \\
\hline concomitant endometrioid carcinoma cases & $14(45.2)$ & \\
\hline TP chemotherapy course & & \\
\hline$\square<6$ & $28(90.3)$ & \\
\hline$\geq 6$ & & \\
\hline & & \\
\hline & & \\
\hline & & \\
\hline & & \\
\hline
\end{tabular}

carcinoma, accounting for $75 \%$ [4], it indicated that endometriosis might undergo probably malignant transformation. Some studies indicated that $40.0 \%$ of ovarian endometrioid carcinoma came from of malignant transformation of ovarian endometriosis, and that the incidence of endometriosis (atypical hyperplasia endometriosis) was $37.5 \%$, twice as much as that reported in other reports. Most of them were confirmed after re-slice and review, indicating that diagnosis level of pathologic doctor is of crucial importance. Pathologic doctors need to improve diagnosis level and enhance collaboration with clinicians to make accurate, comprehensive, and careful pathological evaluation. Lee etc. [5] argued that combination of routine pathological microscopy and immunohistochemical method can diagnose ovarian cancer malignant transformation from endometriosis or primary ovarian endometrioid carcinoma more accurately, which provided sound identifying method. Our study found that there were 18 cases of ovarian endometrioid carcinoma with endometrial cancer, 1 case with mucinous adenocarcinoma, 7 cases with cystadeno carcinoman and 1 case with clear cell carcinoma, indicating that this cancer was more commonly derived from ovary epithelial cell multi-differentiation. Therefore we concluded that the primary pathological manifestation of this cancer was the cancer cell differentiation of ovary germinal epithelium toward endometrium. According to the diagnosis criterion of double primary carcinoma of uterine corpus and the ovary proposed by Scully and Young [6], our data displayed 14 cases of ovarian endometrioid carcinoma combining with endometrioid carcinoma, accounting for $45.2 \%$, indicating ovarian endometrioid carcinoma was easy intercurrent double carcinoma. The main clinical manifestations of the patients with ovarian endometrioid carcinoma were pain and bloating (80.6\%) and vaginal irregular bleeding (54.8\%). Tumor size $>5 \mathrm{~cm}$ by ultrasound examination made up $78.9 \%$. The bigger tumor usually companied with abdominal discomfort, urging the patient to see the doctor. Irregular colporrhagia patients were often required to go to the hospital for check. It made the early stage cancer more easily detected [7]. Consequently, our research shown it was $61.3 \%$ in patients with stage I and II. Early detection provided possibility for early treatment. In the late stage, symptoms such as bloating, abdominal pain, and abnormal vaginal bleeding often appeared. The degree of symptoms was associated with the size and location of the tumor, degree of tumor invasion to nearby organs, and the complications [8].

\section{Treatment}

Our treatment was based on traditional operation combined with post operative chemotherapy. It was reported that the occurrence and development of endometrial adenocarcinoma was likely associated with stimulation of estrogen. It was unknown that the development of ovarian endometrioid carcinoma was likely connected with the stimulation of estrin just as endometrial cancer. How to express oestrogen receptor in ovarian endometrioid carcinoma? However, it is rare on this aspect at present.

Progestin can markedly antagonize on the biological effects of estrogen. Experiment in vitro also verifies progestin's apparent inhibiting effect on the ovarian cancer cell lines proliferation. Progestin has been reported that it would lead to endometrial carcinoma cell differentiation and change of cancerous endometrium secretion phase, significantly reducing mitosis and inhibiting further dysplasia and malignancy, which indicates that progestin has direct inhibition function on tumor cells [9]. However, these researches are still in the initial stage and have not been put into clinical application. We have not given hormone therapy yet. Since all the patients received radiotherapy at other hospitals, the results of radiotherapy were not evaluated. 


\section{Prognosis}

Our research found that there was significant difference between the patient with tumor stage I-II and stage III in the 5-year survival rates. Therefore, early diagnosis and early surgical operation is the key to prognosis improvement. Preoperative ultrasonograph showed that the focus of infection was cystic mixed lesion, highly similar in imaging with ovarian endometrial cysts, indicating the lack of characteristic ultrasonographic manifestation. It can only serve as morphologic index of tumor size instead of histological evidence. So far, CA125 is still the preferred marker of ovarianepithelial carcinomas. This research set 150 $\mathrm{u} / \mathrm{ml}$ as the segmentation point and found that there was no statistical difference between the group of preoperative CA125 $<150 \mathrm{u} / \mathrm{ml}$ and group of CA125 $\geq 150 \mathrm{u} / \mathrm{ml}$ in prognosis, indicating that preoperative CA125 level could only contribute to diagnosis basis but not as prognosis judgment. Platinum-based combination chemotherapy has become the first-line chemotherapeutic regimen for ovarian epithelial carcinomas. One study [10] suggested that the efficacy of TP therapy was superior to other platinum-based chemotherapy. Yet patients did not receive better prognosis after they experienced 6 or more chemotherapy courses, possibly due to the small sample scale of chemotherapy patients with 6 or more chemotherapy courses thus the difference was not reflected accurately. It need further study in larger scale. It was reported that the ovarian endometrioid carcinoma had a 5 -year survival rate of around $40 \%$ to $50 \%$ and had better prognosis than serouscarcinoma or mucous carcinomas [11]. Among patients in this group, 5 -year postoperative survival rate was $54.8 \%$, slightly better than literature. Due to the high pathologic diagnosis requirement, the number of confirmed cases was deficient, avoiding other pathologic type ovarian cancer to be misdiagnosed as endometrioid carcinoma, indicating that strict pathologic diagnosis and distinction of this cancer from ovarian cancer might guarantee a better prognosis for patients. Patients with tumors associated with endometriosis had a higher rate of synchronous endometrial cancer. Cases also demonstrated a lower rate of recurrence and improved 5 year DFS [12].Women with EEOC and concurrent endometriosis showed distinct characteristics and had longer disease-free survival when compared with those without endometriosis [13]. Ovarian endometrioid carcinoma shows great variability in the onset age. In our research, the median age was 52 , and patients less than 55 years old accounted for $61.3 \%$. Our study shown there was no correlation closely between the age of onset and prognosis of ovarian endometrioid carcinoma. The reason might be the small sample size. Hence there was no significant difference. Initial cytoreductive surgery satisfaction degree was closely related to prognosis. Cases of postoperative return of ovarian endometrioid carcinoma within 6 months all died within 5 years. Therefore efforts should be made in perfecting the cytoreductive surgery to improve the prognosis of patients.

\section{Acknowledgements}

We thank Zhang Y and Chang X (Women and children's hospital of Jiaxing university) for their help with this study. This work was supported by Zhejiang clinical medical research foundation (2015-ZYC-A71).

\section{References}

1. Navani SS, Alvarado-Cabrero I, Young RH, Scully RE (1996) Endometrioid carcinoma of the fallopian tube: a clinicopathologic analysis of 26 cases. Gynecol Oncol 63: 371378. [Crossref]

2. Sainz de la Cuesta R, Eichhorn JH, Rice LW, Fuller AF Jr, Nikrui N, et al. (1996) Histologic transformation of benign endometriosis to early epithelial ovarian cancer. Gynecol Oncol 60: 238-244. [Crossref]

3. Erzen M, Rakar S, Klancnik B, Syrjänen K (2001) Endometriosis-associated ovarian carcinoma (EAOC): an entity distinct from other ovarian carcinom as suggested by a nested case-control study. Gynecol Oncol 83: 100-108.

4. Jiang X, Morland SJ, Hitchcock A, Thomas EJ, Campbell IG (1998) Alelotyping of endometriosis with adjacent ovarian carcinoma reveals evidence of a common lineage. Cancer Res 58: 1707-1712.

5. Lee KR, Nucci MR (2003) Ovarianmucinous and mixed epithelial carcinomas of mullerian (endocervica-like) type: a clinic opathologic analysis of four cases of an uncommon variant associated with endometriosis. Int J Gynecol Pathol 22: 42-51. [Crossref]

6. Scully RE.Young RH (1991) Metastatic tumors in the ovary: a problem-oriented approach and review of the recent literature. Semin Diagn Pathol 8: 250-276. [Crossref]

7. Sugiyama T, Kamura T, Kigawa J, Terakawa N, Kikuchi Y, et al. (2000) Clinica characteris $\neg$ tics of clear cell carcinoma of the ovary: a distinct histologic type with poor prognosis and resistance to platinum-based chemotherapy. Cancer 88: 2584-2589. [Crossref]

8. Boyd C, Mc Cluggage WG (2012) Low-grade ovarian serous neoplasms (1ow-grade serous carcinoma and serous borderline tumor) associated with high-grade serous carcinoma or undifferentiated carcinoma: report of a series of cases of an unusual phenomenon. Am J Surg Pathol 36: 368-375. [Crossref]

9. Saegusa M, Okayasu I (1998) Progesterone therapy for endometrial carcinoma reduces cell proliferation but does not alter apoptosis. Cancer 83: 111-121. [Crossref]

10. Ho CM, Chien TY, Shih BY, Huang SH (2003) Evaluation of complete surgica staging with pelvic and para-aortic lymphadenectomy and paclitaxel plus carboplat in chemotherapy for improvement of survival in stage I ovarian clear cell carcinoma. Gynecol Oncol 88: 394-399. [Crossref]

11. Bauknecht T, Birmelin G, Kommoss F (1990) Clinical significance of oncogenes and growth factors in ovarian carcinomas. J Steroid Biochem Mol Biol 37: 855-862. [Crossref]

12. Davis M, Rauh-Hain JA, Andrade C, Boruta DM 2nd, Schorge JO, et al. (2014) Comparison of clinical outcomes of patients with clear cell and endometrioid ovarian cancer associated with endometriosis to papillary serous carcinoma of the ovary. Gynecol Oncol 132: 760-766. [Crossref]

13. Wang S, Qiu L, Lang JH, Shen K, Huang HF, et al. (2013) Prognostic analysis of endometrioid epithelial ovarian cancer with or without endometriosis: A 12-year cohort study of Chinese patients. Am J Obstet Gynecol 209: 566-567. [Crossref]

Copyright: (C2017 Jin XY. This is an open-access article distributed under the terms of the Creative Commons Attribution License, which permits unrestricted use, distribution, and reproduction in any medium, provided the original author and source are credited. 